\title{
Comparative Analysis and Interobserver Variation of Unenhanced Computed Tomography and Intravenous Urography in the Diagnosis of Acute Flank Pain
}

\author{
Abdelmohsen Ben Nakhia ${ }^{a}$ Renu Guptac Adel Al-Hunayan ${ }^{\mathrm{c}}$ Thomas Muttikkal $^{\mathrm{a}}$ \\ Venu Chavan $^{\mathrm{a}}$ Ahmed Mohammed $^{\mathrm{e}}$ Yusuf Ali ${ }^{\mathrm{b}}$ \\ Departments of ${ }^{\mathrm{a}}$ Radiology and ${ }^{\mathrm{b}}$ Urology, Mubarak Al-Kabeer Hospital, and Departments of ${ }^{\mathrm{c}}$ Radiology and \\ d Surgery, and e Center of Medical Education, Faculty of Medicine, Kuwait University, Kuwait
}

\section{Key Words}

Acute flank pain $\cdot$ Interobserver agreement $\cdot$ Intravenous urography $\cdot$ Unenhanced computed tomography

\begin{abstract}
Objectives: The purpose of this study was to compare unenhanced computed tomography (UECT) to intravenous urography (IVU) for detecting urinary tract calculi, signs of obstruction and non-renal causes in the assessment of acute flank pain, and in their interobserver agreement. Patients and Methods: In this prospective study, carried out at a university hospital over a period of 1 year, 36 patients ( 27 males and 9 females) participated. Mean age was $44 \pm 15$ years (range: 14-73 years). The patients presented with acute flank pain and underwent UECT and IVU. The images were blindly evaluated by 2 experienced radiologists and the two techniques compared using the two-tailed McNemar's test for matched pairs; $p$ values $<0.05$ were considered significant. Results: UECT detected stones in 11 (30.6\%) patients, while IVU found them in only 8 (22.2\%). The increased detection by UECT was due to its ability to detect smaller stones $(<6$ $\mathrm{mm}$ ). UECT was also found to be better than IVU in determining calculus position, in detecting primary or secondary signs of obstruction and in identifying non-urinary causes of
\end{abstract}

flank pain. The overall average of agreement, as indicated by kappa values, was 0.88 for UECT and 0.61 for IVU. Conclusion: UECT showed better detectability and interobserver agreement tan IVU, suggesting that UECT could replace IVU as the first imaging modality in the evaluation of acute renal colic.

Copyright $\odot 2010$ S. Karger AG, Basel

\section{Introduction}

Urolithiasis is a common disease in Kuwait with an average annual incidence of hospital admissions of 43.44 per 100,000 population [1]. This rate is almost two times that in England [2]. These patients mainly represent with flank pain [1]. Here, imaging plays an important role in diagnosis as well as management by identifying the size, site and composition of the calculi [3-5].

For several decades, intravenous urography (IVU) has been the primary imaging modality in the evaluation of these patients. Since the introduction of spiral or helical computed tomography (CT) in clinical practice in 1990, many centres have replaced IVU by CT for diagnosing acute renal colic. This is because the size and site of the calculus can be more accurately evaluated by CT than

\section{KARGER}

Fax +4161306 1234

E-Mail karger@karger.ch

www.karger.com (c) 2010 S. Karger AG, Basel

1011-7571/10/0192-0118\$26.00/0

Accessible online at:

www.karger.com/mpp
Prof. Renu Gupta

Department of Radiology, Faculty of Medicine

Kuwait University, PO Box 24923

13110 Safat (Kuwait)

Tel./Fax +965 53304 73, E-Mail renu@hsc.edu.kw 
Table 1. Interobserver agreement for interpretation of UECT results $(n=36)$

\begin{tabular}{|c|c|c|c|c|c|c|c|c|c|c|}
\hline \multirow{2}{*}{$\begin{array}{l}\text { Reader } 1 \\
\text { (T.M.) }\end{array}$} & \multicolumn{10}{|c|}{ Reader 2 (V.C.) } \\
\hline & + & - & + & - & + & - & + & - & + & - \\
\hline+ & 14 & 0 & 13 & 2 & 7 & 2 & 9 & 1 & 5 & 8 \\
\hline- & 0 & 22 & 1 & 20 & 0 & 27 & 1 & 25 & 2 & 21 \\
\hline
\end{tabular}

IVU. CT also gives an approximation of calculus density, predicting the response to treatments like lithotripsy as well as giving an indication of the best follow-up modality [6, 7]. Unenhanced spiral CT (UECT) has shown higher sensitivity, specificity and diagnostic accuracy as compared with IVU in the detection of urinary tract calculi and alternative causes of acute flank pain [8-13].

The purpose of this study was (i) to compare UECT to IVU for detecting urinary tract calculi, signs of obstruction and non-renal causes in the assessment of acute flank pain, and (ii) to investigate whether UECT will enable radiologists to produce more matched consensus reports than IVU by measuring interobserver agreement between 2 experienced radiologists.

\section{Patients and Methods}

This prospective study was carried out on 36 patients, 27 males and 9 females, over a period of 1 year at a university hospital. Mean age was $44 \pm 15$ years (range: $14-73$ years). The patients presented with acute flank pain and clinical diagnosis of urolithiasis and underwent IVU within $24 \mathrm{~h}$ of performing UECT. The study initially included 83 patients, of whom 47 were later excluded as the IVU was not performed within $24 \mathrm{~h}$ of UECT. This was to rule out any change that might occur spontaneously or due to treatment during this interval.

The study was undertaken after obtaining approval from the hospital Ethics Committee. The patients were informed about the study, especially the extra radiation dose, and written consent was obtained. This resulted in recruiting a small number of cases from a total of 1,834 patients who presented with acute flank pain during the study period.

UECT and IVU images were blindly evaluated by 2 experienced specialist radiologists who were blinded to the results of the other technique. The parameters evaluated were the ability to determine the presence, position and size of the calculi, secondary signs of obstruction such as hydroureter, hydronephrosis, nephromegaly, standing of perinephric fat, rim sign, and the detection of non-urinary additional findings [14].

Comparison and Interobserver Variation between CT and IVU
UECT studies were performed using single-slice helical CT, Philips Aura 1.3, Philips Medical System, Nederland BV, The Netherlands. The reconstruction slice thickness was $5 \mathrm{~mm}$. IVU studies were performed using $140 \mathrm{kVp}$ and $100 \mathrm{~mA}$, and the radiographs were taken at $0,5,15$ and 30 min after injection, and immediately after voiding. The contrast medium used was $1 \mathrm{ml} /$ $\mathrm{kg}$ of iohexol $(300 \mathrm{mg} / \mathrm{ml})$. Both procedures were carried out on the same day.

The two techniques were compared using the two-tailed McNemar's test for matched pairs; $p$ values $<0.05$ were considered significant. Measurements of agreement (kappa), which range from -1 to +1 , were calculated for the independent readings made by the 2 radiologists and were considered good when $>0.70$.

\section{Results}

UECT detected stones in 14 patients (table 1), while IVU could detect stones in only 8 (57.1\%). The other 6 patients (table 2), who were found positive for the presence of calculi by UECT, were reported by IVU as being either negative (3 patients) or equivocal (3 patients). Using only the matched consensus findings by the 2 readers for the presence of urinary tract calculi in UECT and IVU, UECT detected stones in 11 (30.6\%) patients (table 3), while IVU detected stones in 8 (22.2\%). The difference between the two modalities, however, was not found to be statistically significant $(p>0.05)$. The increased detectability of UECT was due to its ability to detect smaller stones ( $<6 \mathrm{~mm}$ ) than IVU (6 stones for UECT vs. 1 stone for IVU). UECT was also found to be better than IVU in the determination of calculus position, detection of primary and secondary signs of obstruction, and for identifying non-urinary causes of flank pain. However, this difference between the techniques was significant only for the detection of secondary signs of obstruction $(\mathrm{p}<0.05)$.

Analysis of interobserver agreement (kappa) is shown in table 1 for UECT and in table 2 for IVU. The overall 
Table 2. Interobserver agreement for the presence of stones in IVU $(n=36)$

\begin{tabular}{|c|c|c|c|c|c|c|c|c|c|c|}
\hline \multirow{2}{*}{$\begin{array}{l}\text { Reader } 1 \\
\text { (T.M.) }\end{array}$} & \multicolumn{10}{|c|}{ Reader 2 (V.C.) } \\
\hline & + & - & + & - & + & - & + & - & + & - \\
\hline+ & 8 & 3 & 8 & 3 & 1 & 4 & 2 & 0 & 1 & 0 \\
\hline- & 0 & 25 & 1 & 24 & 1 & 30 & 0 & 34 & 0 & 35 \\
\hline
\end{tabular}

Table 3. Matched consensus findings for the presence of stones in IVU and UECT

\begin{tabular}{|c|c|c|c|c|c|c|c|c|c|c|}
\hline \multirow[t]{2}{*}{ UECT } & \multicolumn{10}{|c|}{ IVU } \\
\hline & \multicolumn{2}{|c|}{ presence of calculus } & \multicolumn{2}{|c|}{ position of calculus } & \multicolumn{2}{|c|}{ primary signs of obstruction } & \multicolumn{2}{|c|}{ secondary signs of obstruction } & \multicolumn{2}{|c|}{ non-urinary pathology } \\
\hline+ & 8 & 3 & 8 & 1 & 1 & 1 & 2 & 7 & 1 & 4 \\
\hline- & 0 & 22 & 0 & 20 & 0 & 27 & 0 & 25 & 0 & 21 \\
\hline
\end{tabular}

${ }^{1} \mathrm{McNemar}$ 's test. Mismatched findings, as reported by the 2 readers, were excluded.

interobserver agreement for UECT (0.77) was comparable to that for IVU (0.75). For the presence and position of calculus, UECT showed better interobserver agreement than IVU (kappa $=1.0$ vs. 0.79 , and 0.83 vs. 0.72 ). For primary signs of obstruction, UECT also showed better agreement than IVU (0.84 vs. 0.22). Furthermore, UECT detected more non-urinary abnormalities than IVU. The latter detected severe spinal degenerative changes, vertebral body fusion and paravertebral calcification in only 1 patient. UECT detected more lesions in 4 patients, e.g. gall stones, fibroid uterus, left adrenal nodular hyperplasia, inflammatory mass of the right iliac fossa and calcified inferior vena cava thrombosis.

\section{Discussion}

In this study, UECT performed better than IVU for the detection of urinary tract calculi. This was not statistically significant, possibly due to the small sample size. These results are consistent with the higher detection capability of UECT over IVU reported previously $[11,12]$. According to these studies, UECT has a sensitivity and specificity of nearly $100 \%$ in evaluating patients with acute flank pain, haematuria or both. Another prospective study comparing UECT and IVU in patients presenting with acute renal colic showed $98.5 \%$ sensitivity for detecting ureteral calculi by UECT as compared to $59 \%$ by IVU $[11,12]$. It was concluded that the diagnosis of ureteric stones could be made confidently by UECT.

UECT showed better agreement values, which ranged between 0.83 and 1.00. The values were better than those reported in another recent study (0.66 and 0.69 for stones on the right and left side; 0.79 and 0.52 for stones in the ureters and the kidney) [15]. The better agreement observed in the present study may be attributed to interpretation of the images by experienced radiologists as well as the multiplanar reformatted reconstruction capability.

The present findings show that in addition to increased detectability and improved interobserver agreement, UECT was more effective in detecting non-renal causes of flank pain; additional non-renal causes of flank pain were found in $13.9 \%$ of the patients on UECT compared to only $5.6 \%$ on IVU. This observation is in line with previous studies which showed that UECT was able 
to diagnose alternate causes of acute flank pain rather than renal calculi in $6-13 \%$ of patients [16-21].

In a study [21] where 1,500 patients presented with acute flank pain, 1,064 (71\%) had alternate or additional findings on UECT. In another study [18] where 1,000 patients underwent unenhanced helical CT for suspected renal colic, $100(10 \%)$ patients had an alternative or additional significant diagnosis, which could be confirmed in $87(8.7 \%)$ patients at follow-up. Detection of ureterolithiasis mimicking alternative abdominal abnormalities by CT in acute settings has a significant impact on patient management [21]. Immediate management is required in cases of pyelonephritis, appendicitis, ovarian torsion, ascending colonic diverticulitis, and acute spontaneous splenic rupture, haemorrhage due to renal arteriovenous malformation, cholecystitis, pancreatitis and aortic dissection $[18,22]$.
The other advantages of UECT over IVU include its ability to detect smaller-size calculi, absence of side effects (renal toxicity, allergic reaction) from intravenous administration of iodine-containing contrast medium, and its speed (5-30 min for UECT vs. 80 to more than $100 \mathrm{~min}$ for IVU). These make UECT a preferred imaging modality as the first-line investigation in acute renal colic.

\section{Conclusion}

UECT seems to be more effective than IVU in the evaluation of acute flank pain with higher interobserver agreement. This, combined with the lack of side effects and time required, makes UECT better suited than IVU as the first imaging modality for the evaluation of acute renal colic.

\section{References}

-1 Al-Hunayan A, Abdul-Halim H, Kehinde EO, Al-Awadi K, El Barky E, Al-Ateequi A: Mode of presentation and first line of management of non-recurrent urolithiasis in $\mathrm{Ku}$ wait. Int J Urol 2004;11:963-968.

- El-Reshaid K, Mughal H, Kapoor M: Epidemiological profile, mineral metabolic pattern and crystallographic analysis of urolithiasis in Kuwait. Eur J Epidemiol 1997;13: 229-234.

-3 Singal RK, Denstedt JD: Contemporary management of ureteral stones. Urol Clin North Am 1997;24:59-70.

4 Marberger M, Holblauer J, Turk C, Hobarth K, Albretch W: Management of ureteric stones. Eur Urol 1994;25:265-272.

5 Hubner WA, Irby P, Stoller M: Natural history and current concepts for the treatment of small ureteral calculi. Eur Urol 1993;24: 172-176.

6 Olcott EW, Sommer FG, Napel S: Accuracy of detection and measurement of renal calculi: in vitro comparison of three-dimensional spiral CT, radiography and nephrotomography. Radiology 1997;204:19-25.

7 Sommer FG, Jeffrey RB Jr, Rubin GD, Napel S, Rimmer SA, Benford J, Harter PM: Detection of ureteral calculi in patients with suspected renal colic: value of reformatted noncontrast helical CT. AJR Am J Roentgenol 1995; 165:509-513.

8 Greenwell TJ, Woodhams S, Denton ER, MacKenzie A, Rankin SC, Popert R: One year's clinical experience with unenhanced spiral computed tomography for the assessment of acute loin pain suggestive of renal colic. BJU Int 2000;85:632-636.
9 Heidenreich A, Desgrandschamps F, Terrier F: Modern approach of diagnosis and management of acute flank pain: review of all imaging modalities. Eur Urol 2002;41:351362.

10 Mendelson RM, Arnold-Reed DE, Kuan M, Wedderburn AW, Anderson JE, Sweetman G, Bulsara MK, Mander J: Renal colic: a prospective evaluation of non-enhanced spiral CT versus intravenous pyelography. Australas Radiol 2003;47:22-28.

11 Fielding JR, Silverman SG, Samuel S, Zou $\mathrm{KH}$, Loughlin KR: Unenhanced helical CT of ureteral stones: a replacement for excretory urography in planning treatment. AJR Am J Roentgenol 1998;171:1051-1053.

12 Katz DS, Lane MJ, Sommer FG: Unenhanced helical CT of ureteral stones: incidence of associated urinary tract findings. AJR Am J Roentgenol 1996;166:1319-1322.

13 Preminger GM, Vieweg J, Leder RA, Nelson RC: Urolithiasis: detection and management with unenhanced spiral CT. Radiology 1998; 207:308-309.

14 Boulay I, Holtz P, Foley WD, White B, Begun F: Ureteral calculi: diagnosis efficacy of helical CT and implications for treatment of patients. AJR Am J Roentgenol 1999;172:14851490.

15 Lund L, Larsen UL, Anderson E, Mikkelsen NT, Holt G: The outcome of computed tomography in patients with acute renal colic from a low-volume hospital. Int Urol Nephrol 2008;40:255-258.
6 Smith RC, Rosenfield AT, Choe KA, Essenmacher KR, Verga M, Glickman MG, Lange RC: Acute flank pain: comparison of noncontrast-enhanced CT and intravenous urography. Radiology 1995;194:789-794

17 Thomson JM, Glocer J, Abbott C, Maling TM, Mark S: Computed tomography versus intravenous urography in diagnosis of acute flank pain from urolithiasis: a randomised study comparing imaging costs and radiation dose. Australas Radiolol 2001;45:291-297.

18 Katz DS, Scheer M, Lumerman JH, Mellinger BC, Stillman CA, Lane MJ: Alternative or additional diagnoses on unenhanced helical computed tomography for suspected renal colic: experience with 1,000 consecutive examinations. Urology 2000;56:53-57.

19 Dalrymple NC, Verga M, Anderson KR, Bove P, Covey AM, Rosenfield AT, Smith RC: The value of unenhanced helical computerized tomography in the management of acute flank pain. J Urol 1998;159:735-740.

20 Chen MY, Zagoria RJ: Can noncontrast helical computed tomography replace intravenous urography for evaluation of patients with acute urinary tract colic? J Emerg Med 1999;17:299-303.

21 Hoppe H, Studer R, Kessler TM, Vock P, Studer UE, Thoeny HC: Alternative or additional findings to stone disease on unenhanced computerised tomography for acute flank pain can impact management. J Urol 2006;175:1725-1730.

22 Rucker CM, Menias CO, Bhalla S: Mimics of renal colic: alternative diagnoses at unenhanced helical CT. Radiographics 2004;24: S11-S33. 